Volume 9, No.5, September - October 2020

International Journal of Advanced Trends in Computer Science and Engineering

Available Online at http://www.warse.org/IJATCSE/static/pdf/file/ijatcse01952020.pdf

https://doi.org/10.30534/ijatcse/2020/01952020

\title{
A Comparison of Classification Methods to Classify the Breast Lesions from Ultrasound Images using Texture Feature Extraction
}

\author{
Telagarapu Prabhakar ${ }^{1^{*}}, \mathrm{~S}$ Poonguzhali ${ }^{2}$ \\ ${ }^{1}$ Department of Electronics and Communication Engineering, GMR Institute of Technology, Rajam, AP, India \\ prabhakar.t@gmrit.edu.in \\ ${ }^{2}$ Department of Electronics and Communication Engineering, CEG, Anna University, India
}

\begin{abstract}
From past few decades there is huge death rates due to breast cancer. Detection of breast cancer at first stage itself is very important. For detection and screening Mammography technique is usd in hospitals. But it was not comfortable for many young women because it involves radiation during testing. The advantage of Ultrasound over mammography is its non-invasive procedure, no radiation like mammography and it is available with less cost. However, the excellence of ultrasound image is corrupted by a speckle noise. In this paper we are developing an algorithm which will reduce the speckle noise. We also proposed some Feature Extraction and classification methods for tumor detection from ultrasound breast images.
\end{abstract}

Key words: Breast cancer, Ultrasound Image, GLCM, Wavelet, Curvelet; Contourlet, SVM and BPN.

\section{INTRODUCTION}

Now a days Breast cancer is very common for most of the women's [1] and it is rare case for the men. For detection and screening Mammography technique was used. But it has many disadvantages. Young ladies have dense breast, so it causes pain to the patients while screening, for them this method is not suitable. So many women's preferring Ultrasound technology because of its non-invasive procedure, no radiation like mammography and it is available with less cost. But the images produced by an ultrasound technology was degraded because of speckle noise there by a human observer cannot find the details regarding diagnostic. Many experiments were implemented for reducing the effect of speckle noise from ultrasound images as well as no. of architectures regarding filters were discussed here [2]. J. S. Lee used mean and median filters for reduction of speckle noise [3]. V. S. Frost et.al used Lee filter he got improvement compared to previous filters [4], Y. Yu et.al used the Frost Filter for reduction of noise [5], and Renjie Liao eReceived the paper. WE will reply you soont.al used the SRAD Filter [6]. All these removal methods are known as "feature preserving" and "edge preserving" filters but it exists some limitations in these methods. The first limitation were these filters are mainly depending upon the window shape and size. It will not accept any changes other than prescribed point. Already we know that window filter is large compared to the object there by it causes blurring as well as edges will be smoothened. Thereby A minor window will reduce the effect of speckle noise and smoothing process in the filter. Following next limitation is in these methods there is no process involved like enhancement of the edges, but it will only reduce smoothing of the edges. So, noise will be present at neighborhood of an edge. Third limitation is all the discussed methods are non-directional. Therefore, for the next process of an image, without changing the features of an image noise will be reduced. The important technique was discussed in Image texture analysis. It uses gray level properties of an image for expressing the non-morphological features [7]. Here we applied proper multi-resolution transform, and from transformed image we may extract the texture features. These features are mainly used for characterized the properties of the texture and it will also used for training the classifier for identification of the texture class. Textural variation is the useful feature in ultrasound image which is used for identifying the benign tumors and malignant tumors. Here the algorithm which can be used for many features. Manually there will be a difficult to check and pick up the best features. So, they proposed principle component analysis [8]. The Multiresolution Analysis tool such as Wavelet, Ridgelet, and features from Co-occurrence-based methods are computed for extraction of the features and for feature selection a genetic algorithm is used for better classification. Features from various methods are extracted and classification of variations from the normal is compared by utilizing BPN for recognition rate. SVM network and many more methods belongs to $\mathrm{ML}$ are proposed. it is divided into benign tumor or malignant tumor as per the features [9]. In this paper, the ultrasound image is denoised by Median, Mean, Weiner, SRAD (Speckle reduction anisotropic diffusion) filters and wavelet thresholding method. After denoising, the GLCM features for the benign and malignant tumor are extracted by using feature extraction methods such as GLCM, Wavelet, 
Curvelet, and Contourlet. Features are divided into the benign tumor or malignant tumor using the SVM and BPN network and best feature extraction and classification methods are evaluated.

\section{METHODOLOGY}

In this paper, ultrasound images of the breast are taken from GE Healthcare LOGIQ E9 ultrasound scanner, by using the linear transducer array having $5 \mathrm{MHz}$ frequency and image size is $800 \times 600$ pixels. The algorithms are applied to sub-images of both benign and malignant masses of breast. The proposed dataset contains 60 cases, out of that 30 cases comes under benign masses and remaining 30 belongs to malignant are solid masses. Here by using different methods the images are prepressed for eliminating the speckle noise. After speckle removal, the GLCM features are extracted from both benign and malignant tumor regions by several feature extraction methods and classified as benign or malignant. Here we are using GLCM, Wavelet transform, Curvelet transform and Contourlet transform. The extracted features are classified with SVM, and BPN techniques to distinguish malignant tumor from benign. The complete flow of the efficient texture analysis with transforms and from where GLCM is extracted to classify the breast tumor from ultrasound images is shown in Figure 1.

\subsection{Preprocessing}

As we know that it is a multiplicative noise, and directly proportional to the value of pixel intensity. With the effect of noise image will be degraded. So that degraded image is represented by using the below expression

$$
G=I * N
$$

Here G, I and $\mathrm{N}$ are the function of $\mathrm{x}, \mathrm{y}$. Where $\mathrm{G}$ represents degraded image, I represent the original image and $\mathrm{N}$ represents noise multiplication of that image. We have used various filters like mean, median, wiener; Speckle reduction anisotropic Diffusion filter (SRAD) and wavelet thresholding techniques for reducing the noise in an image. The PSNR has been found for all the filters. Every image is processed with the best filter for further classification.

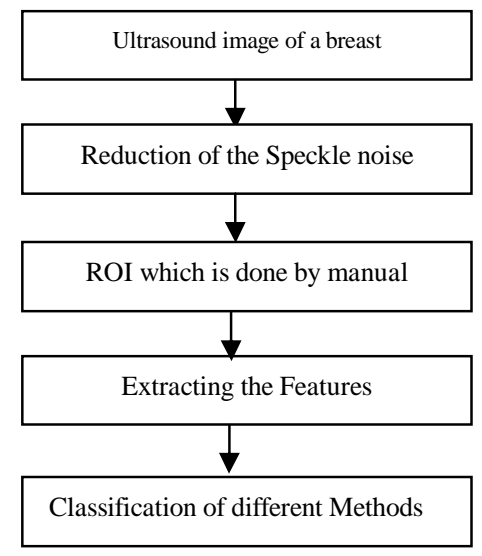

Figure 1: Texture analysis with transforms and from where GLCM is extracted to classify the breast tumor from ultrasound images

\subsection{Feature Extraction Methods}

Extraction of the features having main role in pattern classification. The features are extracted for every $10 \mathrm{X} 10$ pixel of the 64X64 slice of the tumor by various feature extraction methods and classified as a benign or malignant tumor. The region of interest is manually cropped. The GLCM features are obtained by all the transforms, and by using feature extraction methods we can extract the features which are used for classifications. With GLCM method matrix-based features are extracted, and these are mainly used for measuring the texture in images. These are 2-D histograms. An element of the GLCM is denoted with P (i, j, $d, \theta$ ) where $i$ and $j$ are the gray levels joint probability having a space $d$ in $\theta$ direction. For simplification of the process the values of $\theta$ can be taken as multiples of 45 degrees and here distance is also known as Manhattan distance. The features like Autocorrelation, Dissimilarity, Cluster Prominence, and Cluster Shade etc as per our requirement [9].

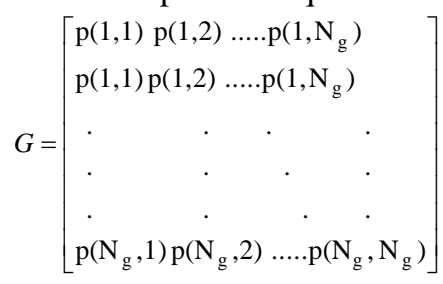

$P(x, y)$ is $(x, y)^{t h}$ in spatial dependence matrix having a normalized gray tone. $\mathrm{N}_{\mathrm{g}}$ represents quantized image having different grey levels

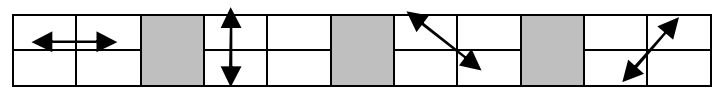

Figure 2: For calculating Haralick texture features we are using Four directions of adjacency. Mainly these statistics are calculated for co-occurrence matrices which are generated by the directions of adjacency.

\begin{tabular}{|l|l|l|}
\hline $\mathrm{LL}^{2}$ & $\mathrm{LH}^{2}$ & \multirow{2}{*}{$\mathrm{LH}^{1}$} \\
\cline { 1 - 2 } $\mathrm{HL}^{2}$ & $\mathrm{HH}^{2}$ & \\
\hline $\mathrm{HL}^{1}$ & & $\mathrm{HH}^{1}$ \\
\hline
\end{tabular}

Figure 3: Two-Level Image decomposition by using DWT

Here we are applying 2D-DWT by using the wavelet and resolution levels which are used for yielding the coefficient matrices in detailed as well as for approximation. At each level, for getting detailed information we are using HP filter and for getting coarse approximation we are using the combination of LP filter and scaling function [11]. The GLCM features are extracted by this method and given to the classifier. 
Curvelet Transform is an enhanced variant of the Ridgelet. It tells to bend singularities substantially more proficient than the ridgelet. Curvelet has better directional qualities. Here the coefficients by Curvelets are taken from sporadically sampling the Fourier coefficients of an image. By observing we can say that here there is no loss of information because it is covered with frequency spectrum. The GLCM features are extracted for the lesion by utilizing Curvelet transform. These features are then given to the classifier. Do and Vetterli proposed this concept and it represents directional multiresolution image. For obtaining features regarding typical images such as smooth contours we are using double filter bank. It contains mainly LP and DFB. For detection of point discontinuities in an image LP is used and for mapping the point discontinuities into linear structures DFB is used. The GLCM features are extracted for the tumor by utilizing Contourlet transform decomposition. These features are then given to the classifier. Based upon statistical approach the SVM is presented by Vapnik. It determines hyperplane that can isolate the input images. sometimes it cannot be isolated by a hyperplane, so, SVM is used for converting it into a feature space having higher dimension by utilizing the kernel function. We have many types of kernels in use. In this paper, we used linear kernel. The Linear kernel is the least difficult kernel function.

$$
S(x, y)=x^{T} y+c
$$

Assume $S$ contains n labeled samples (x1, y1) ........ (xn, $\mathrm{yn})$. where $\mathrm{S}$ is a trainer set. ' $\mathrm{C}$ ' is steady parameter it will manage the gap between high and less margins [17]. Back-propagation algorithms have been ordinarily utilized for the training of the artificial neural network. In this procedure we utilized 4 hidden neurons and weights between the processing units are iteratively balanced so that the general error measure is reduced.

\section{RESULTS AND DISCUSSION}

\subsection{Speckle Reduction}

Ultrasound Images are denoised by using different filtering techniques. The outputs of the filters are shown in Figure: 4. PSNR is designed with different filtering techniques. In this process ground truth is obtained by the radiologist. The Table 1 show that the PSNR value for the wavelet thresholding is 73.25 which is best compared to discussed filter techniques. Thus, this technique further considered for this work to remove the speckle noise.

Table 1: Comparison of filter Techniques with PSNR Values

\begin{tabular}{|l|c|}
\hline Filters & $\begin{array}{l}\text { Denoised Images } \\
\text { PSNR values in dB }\end{array}$ \\
\hline $\begin{array}{l}\text { Wavelet } \\
\text { Thresholding }\end{array}$ & 73.25 \\
\hline SRAD & 70.33 \\
\hline Wiener & 24.45 \\
\hline Mean & 23.60 \\
\hline Median & 22.01 \\
\hline
\end{tabular}

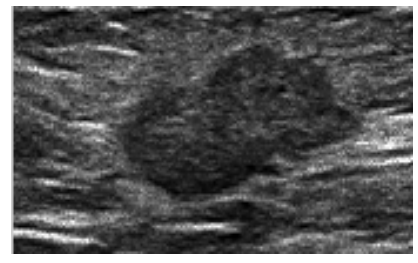

(a) Input Image

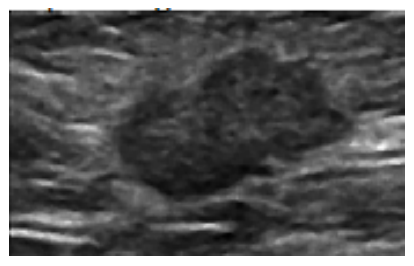

(b) Median filter

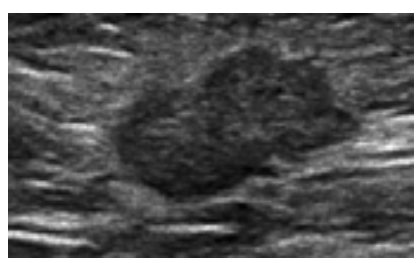

(c) Mean filter

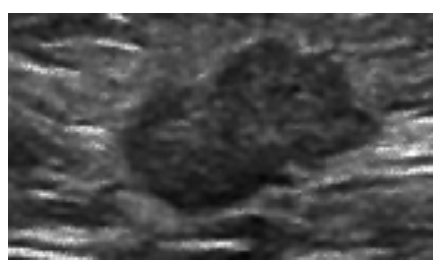

(d) Wiener filter

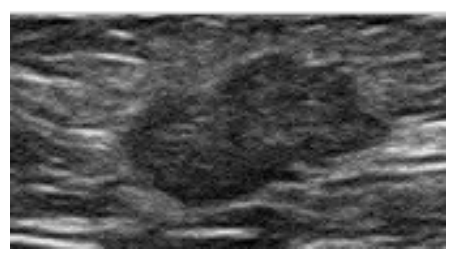

(e) SRAD filter

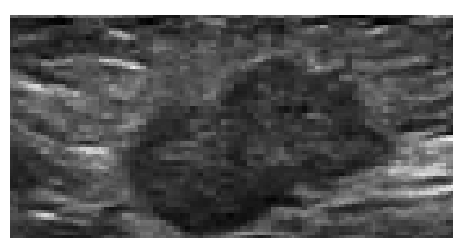

Figure 4: Denoised images

\subsection{Feature Extraction}

The GLCM features for both benign and malignant tumor are extracted from denoised images and sub images of wavelet, curvelet, contourlet transforms. The GLCM based upon many features like Autocorrelation, Dissimilarity, Cluster Prominence, and Cluster Shade etc. 


\subsection{Classification}

From benign and malignant total 60 images are taken in that 40 image samples with 15 features are used as training data set. 20 image samples with 15 features are used as a testing data set. 20 benign and 20 malignant images are considered for training and remaining 10 from each category images are considered for testing. We are giving extracted features as input to the classifiers such as SVM and BPN. For all the four feature extraction methods we measured the values of accuracy, sensitivity, and specificity by using SVM and BPN classifiers are shown in Table 2. The classification accuracy using curvelet features is higher when compare GLCM and Wavelet feature extraction. For curvelet features, both SVM and BPN performances both are same as wavelet. The accuracy of BPN with contourlet feature extraction is $70 \%$ and $80 \%$ for SVM. The SVM performance dominates than BPN with contourlet features. The overall the performance of contourlet transform method with SVM provides the better accuracy over the other feature extraction methods. Some features may create a problem regarding classification. If we are selecting main features by feature selection, then the performance of the classifier may be improved for better diagnosis. The overall performance of SVM method is higher than BPN. The advantage of SVM over BPN is less computational time. The time required for training BPN could be longer, as the size of hidden neurons increases. Thus, the given method can classify benign and malignant with respect to the three objective indices more accurately.

Table2: Comparison of Classification Techniques

\begin{tabular}{|l|c|c|c|c|c|c|}
\hline \multirow{2}{*}{$\begin{array}{l}\text { Feature } \\
\text { extraction } \\
\text { method }\end{array}$} & \multicolumn{2}{|c|}{$\begin{array}{c}\text { Accuracy } \\
(\%)\end{array}$} & \multicolumn{2}{c|}{$\begin{array}{c}\text { Sensitivity } \\
(\%)\end{array}$} & \multicolumn{2}{c|}{$\begin{array}{c}\text { Specificity } \\
(\%)\end{array}$} \\
\cline { 2 - 7 } & SVM & BPN & SVM & BPN & SVM & BPN \\
\hline GLCM & 75 & 70 & 90 & 70 & 60 & 70 \\
\hline Wavelet & 70 & 70 & 60 & 80 & 80 & 60 \\
\hline Curvelet & 75 & 75 & 70 & 70 & 80 & 80 \\
\hline Contourlet & 80 & 70 & 80 & 60 & 80 & 80 \\
\hline
\end{tabular}

$$
\begin{aligned}
& \text { Accuracy }=\frac{T P+T N}{T P+T N+F P+F N} \\
& \text { Sensitivity }=\frac{T P}{T P+F N} \\
& \text { Specificity }=\frac{T N}{T N+F P}
\end{aligned}
$$

TP indicates Malignant classified correctly as Malignant TN indicates Benign classified correctly as Benign FP indicates Benign classified incorrectly as Malignant FN indicates Malignant classified incorrectly as Benign

\section{CONCLUSION}

The proposed contourlet feature extraction method with SVM classification produces a high classification rate $(80 \%)$. If the main features are selected, then the classifier performance may be improved for better diagnosis. The features are extracted by using GLCM, Wavelet transform, Curvelet Transform and Contourlet Transform Methods. The GLCM based contourlet transform gives a better result than GLCM, wavelet and curvelet transform for feature extraction. The extracted features are given to the classifiers such as SVM and BPN. The performance of classification using SVM method is higher than BPN for contourlet features. Therefore, the proposed method accurately detects breast cancer which will be very useful for the patients.

\section{REFERENCES}

1. Siegel et.al. Cancer statistics, 2013, CA: a cancer journal for clinicians" 63, no. 1 (2013): 11-30.

2. Zhang, Ju, and Yun Cheng. Despeckle Filters for Medical Ultrasound Images, In Despeckling Methods for Medical Ultrasound Images, pp. 19-45. Springer, Singapore, 2020.

3. Yu, Yongjian, and Scott T. Acton. Speckle reducing anisotropic diffusion, IEEE Transactions on image processing 11, no. 11 (2002): 1260-1270.

4. Chand, Satish. A comparative study of breast cancer tumor classification by classical machine learning methods and deep learning method, Machine Vision and Applications 31, no. 6 (2020): 1-10..

5. Haralick, Robert M., Karthikeyan Shanmugam, and Its' Hak Dinstein. Textural features for image classification, IEEE Transactions on systems, man, and cybernetics 6 (1973): 610-621..

6. Donoho, David L. De-noising by soft-thresholding, IEEE transactions on information theory 41 , no. 3 (1995): 613-627..

7. Candes, Emmanuel, Laurent Demanet, David Donoho, and Lexing Ying. Fast discrete curvelet transforms, Multiscale Modeling \& Simulation 5, no. 3 (2006): 861-899.

8. Do, Minh N., and Martin Vetterli. The contourlet transform: an efficient directional multiresolution image representation, IEEE Transactions on image processing 14, no. 12 (2005): 2091-2106.

9. V.N. Vapnik, Statistical Learning Theory, John Wiley and Sons, New York (1998). 